\title{
Divertículo de Zenker: manejo endoscópico con o sin diverticulotomo
}

\section{Zenker's diverticulum: endoscopic treatment with or without diverticulotome}

\author{
Martín Alonso Gómez, ${ }^{*}$ (D) Óscar Fernando Ruíz, ${ }^{2}$ [C] Hernando Marulanda. ${ }^{3}$ 두
}

\begin{tabular}{|c|}
\hline $\begin{array}{l}\text { GACCESO ABIERTO } \\
\text { Citación: } \\
\text { Gómez MA, Ruíz OF, Marulanda H. Divertículo } \\
\text { de Zenker: manejo endoscópico con o sin } \\
\text { diverticulotomo. Rev Colomb Gastroenterol. } \\
\text { 2020;35(4):421-429. https://doi. } \\
\text { org/10.22516/25007440.504 }\end{array}$ \\
\hline $\begin{array}{l}1 \text { Médico Internista y Gastroenterólogo. Unidad } \\
\text { de Gastroenterología y Ecoendoscopia (UGEC). } \\
\text { Hospital Universitario Nacional de Colombia. } \\
\text { Profesor asociado de Medicina, Universidad } \\
\text { Nacional de Colombia. Bogotá, Colombia. } \\
2 \text { Médico Internista y Gastroenterólogo. Unidad } \\
\text { de Gastroenterología y Ecoendoscopia (UGEC). } \\
\text { Hospital Universitario Nacional de Colombia. } \\
\text { Bogotá, Colombia. } \\
\text { 3 Médico Internista y Gastroenterólogo, } \\
\text { Universidad Nacional de Colombia. Hospital } \\
\text { Universitario Nacional de Colombia. Bogotá, } \\
\text { Colombia. }\end{array}$ \\
\hline $\begin{array}{l}\text { *Correspondencia: Martín Alonso Gómez } \\
\text { martinalonsogomezz@gmail.com }\end{array}$ \\
\hline $\begin{array}{l}\text { Fecha recibido: } \quad 03 / 02 / 20 \\
\text { Fecha aceptado: } 24 / 08 / 20\end{array}$ \\
\hline
\end{tabular}

\begin{abstract}
Resumen
El divertículo de Zenker se define como un divertículo localizado en la pared esofágica posterior que tiene como cuello proximal al músculo cricofaríngeo. La mayoría de los pacientes presenta síntomas después de los 60 años de edad. Antes el manejo estándar se realizaba la diverticulotomía quirúrgica, pero desde hace más de 20 años se introdujo el manejo endoscópico. Desde hace más de 15 años se introdujo un diverticulotomo que facilita la realización del procedimiento, pero su uso no se ha instaurado ampliamente en Colombia. Por ello, el objetivo de este estudio es evaluar si la diverticulotomía endoscópica con el uso de este dispositivo es más fácil (rápida) que el método estándar. Materiales y métodos: se realizó un estudio prospectivo evaluando dos métodos endoscópicos para tratar el divertículo de Zenker, con el objetivo principal de conocer en cuál de ellos el tiempo de incisión del tabique era menor. El tiempo del procedimiento se contaba después de la colocación de la sonda en el método estándar o después de colocar el diverticulotomo en posición (método con diverticulotomo), ya que es el momento exacto en el cual se inicia el corte del tabique y se contabilizaba el tiempo (registro en video) cuando este corte terminaba. Resultados: de enero de 2015 a diciembre de 2018 en total se incluyeron 20 pacientes. El diagnóstico de Zenker fue realizado por una endoscopia y esofagograma para clasificar el tamaño del mismo. El promedio de edad de los pacientes fue de 61 años (46-85). En cada uno de los grupos se asignaron 10 pacientes. En 7 pacientes del grupo método estándar se resolvieron los síntomas, en comparación con 9 del grupo del método con diverticulotomo. Los tres pacientes que no respondieron presentaron en el seguimiento a 18 meses un estadio II de la escala de deglución de resultados funcionales. El tiempo promedio del procedimiento fue de 32 (25-45) minutos en el grupo del método estándar y 12 (7-15) minutos en el grupo de método con diverticulotomo $(p<0,001)$. Conclusiones: La miotomía del divertículo de Zenker con el uso de endoscopia flexible utilizando el diverticulotomo tiene beneficios potenciales en términos de tiempos operatorios más cortos, estancias posoperatorias más cortas y un inicio más temprano de la dieta. Desde el punto de vista técnico, permite una mejor exposición del tabique, lo que facilita su corte, pero se necesitan estudios más grandes para confirmar estos resultados.
\end{abstract}

Palabras claves

Disfagia, Zenker, diverticulotomía endoscópica, diverticulotomo, complicaciones.

\section{INTRODUCCIÓN}

El divertículo de Zenker es un divertículo por pulsión que, dada su ubicación crítica, genera disfagia en el paciente, lo cual disminuye significativamente su calidad de vida debido a que impide la adecuada alimentación y, además, genera diferentes síntomas como regurgitación, tos crónica y pérdida de peso, y acarrea otras complicaciones, de las cuales se considera que la más grave es la broncoaspiración recurrente, que produce neumonías a repetición y, a su vez, produce una alta tasa de morbimortalidad (1). La región anatómica donde se genera se denomina triángulo 


\begin{abstract}
Zenker's diverticulum is defined as a diverticulum located in the posterior esophageal wall, just above the cricopharyngeal muscle. Most patients experience symptoms in this area after the age of 60 . In the past, the standard treatment was surgical diverticulotomy, but endoscopic treatment was introduced over 20 years ago. For more than 15 years, overtubes are introduced to facilitate the procedure, but their use is not been widely established in our country. Therefore, this study aims to evaluate whether endoscopic diverticulotomy with the use of this device is easier (faster) than the standard method. Materials and methods: A prospective study was conducted to establish which of the two endoscopic methods to treat Zenker's diverticulum (ZD) made the septum incision time shorter. The time count was done using video recording and started when the probe - standard method (SM) — or the overtube (MD) were placed, as this is the exact moment when septum cutting begins. The count stopped when the cut was finalized. Results: A total of 20 patients were treated from January 2015 to December 2018. Zenker's diverticulum diagnosis was achieved by endoscopy and esophagogram to classify the size of the tumor. The average age of the patients was 61 years (46-85). Ten patients were assigned to each group. Symptoms resolved in 7 patients of the SM group, compared to 9 in the MD group. The three patients who did not respond to the treatment were classified as stage II in the functional outcome swallowing scale at 18 months of follow-up. The average time of the procedure was $32(25-45)$ minutes in the SM group and $12(7-15)$ minutes in the MD group $(p<0.001)$. Conclusions: ZD myotomy with flexible endoscopy using an overtube has potential benefits in terms of shorter operative times, shorter post-operative stays, and an earlier reintroduction of food. From a technical point of view, this method allows for better exposure of the septum, which facilitates cutting. However, more extensive studies are needed to confirm these results.
\end{abstract}

Keywords

Dysphagia, Zenker, endoscopic diverticulotomy, diverticulotome, complications.

de Killian, se localiza en la pared posterior de la hipofaringe, entre el músculo constrictor faríngeo inferior y las fibras transversas del cricofaríngeo (Figura 1) (2). El divertículo de Zenker toma su nombre del patólogo alemán Friedrich Albert Von Zenker, quien, junto con Von Ziemssen, en 1878 reportó 23 casos en la Universidad de Erlangen y lo definió como "una formación herniaria de la membrana hipofaríngea posterior, proximal al esfínter esofágico superior". Sin embargo, debe reconocerse que fue descrito por primera vez no por el Dr Zenker sino por el Dr. Abraham Ludlow en 1769 (3, 4).

Desde el punto de vista fisiopatológico, es un divertículo por pulsión; su origen se debe al aumento de la presión intraluminal que lleva a un prolapso de la mucosa y submucosa (por lo que se considera un pseudodivertículo), en el área del triángulo de Killian, donde se encuentra un tono mural normalmente bajo, asociado con presiones relativamente reducidas en el espacio retrofaríngeo contiguo, que conduce a la formación del divertículo en la parte posterior (Figura 1). Sin embargo, aún no es claro cuáles son las fuerzas exactas que contribuyen al aumento de las presiones luminales, por lo que se considera un desafío la medición manométrica por la asimetría de la presión del esfínter y su amplio rango de movimiento $(5,6)$. El divertículo de Zenker se considera un trastorno infrecuente (tasas de ocurrencia entre el 0,01\% y el 0,11\% de la población

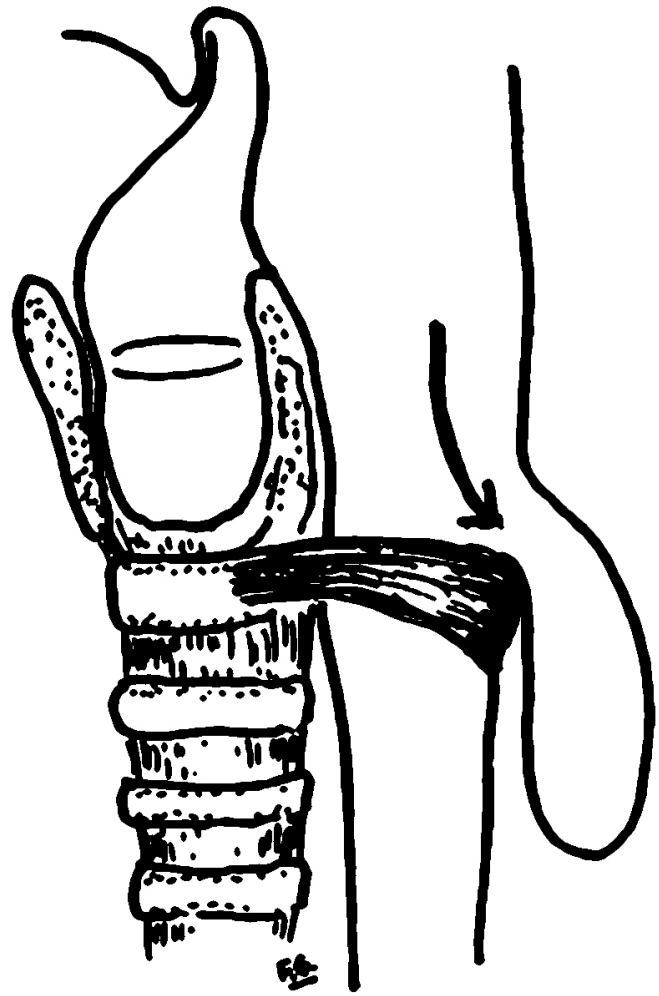

Figura 1. Representación anatómica del divertículo de Zenker. Tomada de referencia (4) 
general), aunque la verdadera incidencia es difícil de determinar debido a diferencias en la gravedad de los síntomas, pero es más frecuente en hombres entre la séptima y octava décadas de la vida $(6,7)$. La disfagia es el síntoma cardinal y se conocen 2 mecanismos causales: apertura incompleta del esfínter esofágico superior y compresión extrínseca del esófago cervical por el propio divertículo. Desde el punto de vista clínico, el borborigmo cervical, particularmente en presencia de un bulto palpable en el cuello, es casi patognomónico de divertículo de Zenker, aunque no es un hallazgo frecuente (8). Los signos de alarma como el dolor local y la hemoptisis o hematemesis pueden indicar ulceración o carcinoma de células escamosas dentro del divertículo de Zenker, que tiene una incidencia de $0,4 \%$ a $1,5 \%(6,9)$.

La videofluoroscopia con trago de bario es útil para el diagnóstico: proporciona información sobre el tamaño y la ubicación, y en ocasiones puede identificar lesiones mucosas en el saco diverticular $(10,11)$; además, puede realizar el diagnóstico diferencial del divertículo de Killian-Jamieson, menos común y más pequeño, que surge en el esófago cervical proximal anterolateral y es inferior al músculo cricofaríngeo (12). Aunque útil, la videofluoroscopia con trago de bario tiene un rendimiento diagnóstico que está condicionado por las variaciones en la calidad en la realización y la interpretación de estos estudios. La endoscopia digestiva alta es necesaria para descartar la presencia del carcinoma escamocelular (13) y es esencial para definir la anatomía y, en consecuencia, orientar la estrategia terapéutica.

Se han propuesto varios sistemas de clasificación que tienen como base el tamaño y la morfología, incluidos los sistemas Brombart (Tabla 1) (14) y Morton/Bartley (Tabla 2) (15).

Tabla 1. Clasificación de Brombart (tamaño y morfología de los divertículos) (14)

Etapa

I Eje longitudinal 2-3 mm, divertículo en forma de espina de rosa

II Eje longitudinal de 7-8 mm, divertículo en forma de palo

III Eje orientado en sentido caudal de $>1 \mathrm{~cm}$ de longitud

IV Compresión del esófago

Tomado de: Mantsopoulos K et al. Eur Arch Otorhinolaryngol. 2014;271(3):583-8.

El tratamiento quirúrgico se ha descrito desde el siglo XIX, con una orientación similar al tratamiento de la hernia. Solo en las últimas décadas se introdujo la miotomía cricofaríngea y, más recientemente, la septotomía con técnica endoscópica ha sido aceptada como una técnica de vital importancia en la corrección del divertículo de Zenker, demostrando la mejoría en relación con la acumulación de alimentos (líquidos y sólidos) y la supresión de todos o la gran mayoría de los síntomas (15). Sin embargo, tanto los abordajes transcervicales abiertos como los endoscópicos están asociados con complicaciones y riesgos potenciales, que son menos frecuentes en la técnica endoscópica, por lo que actualmente es la primera opción, considerando que estos pacientes se presentan más frecuentemente desnutridos, con una edad avanzada y casi siempre con comorbilidades asociadas, lo que aumenta exponencialmente el riesgo quirúrgico (16-18).

Tabla 2. Clasificación de Morton/Bartley (tamaño) (15)

\begin{tabular}{|cc|}
\hline Etapa & Definición \\
\hline I & $<2 \mathrm{~cm}$ \\
II & De $2-4 \mathrm{~cm}$ \\
\hline$I I$ & $>4 \mathrm{~cm}$ \\
\hline
\end{tabular}

Tomado de: Miller FR, Taylor C. Zenker's Diverticulum: Endoscopic Surgical Management Options. Clin Surg. 2017;2:1422.

\section{MATERIALES Y MÉTODOS}

Se realizó un estudio prospectivo evaluando dos métodos endoscópicos para tratar el divertículo de Zenker, con el objetivo principal de conocer en cuál de ellos el tiempo de incisión del tabique era menor, lo que se traduce en un procedimiento más corto (tiempo quirúrgico y anestésico) y también se reduce el riesgo de morbimortalidad relacionada con el mismo.

Los objetivos secundarios fueron la resolución de los síntomas de acuerdo con la escala de resultados funcionales de deglución (FOSS), evaluada antes y después del procedimiento; y la resolución de las complicaciones mayores definidas como sangrado (que requiera soporte transfusional o reintervención endoscópica para su control), perforación y recurrencia de síntomas con un seguimiento promedio de 18 meses.

Los pacientes enviados para diverticulotomía eran evaluados clínicamente, incluyendo la presencia de disfagia para sólidos o líquidos, tos, regurgitación, globo histérico, broncoaspiración, pérdida de peso y necesidad de nutrición suplementaria, entre otros. Se determinó una puntuación FOSS antes y después del procedimiento utilizando la metodología para calcular la puntuación descrita por Salassa (Tabla 3) (19, 20). Se tomaron en cuenta solo los pacientes en estadios II, III, IV y V de la escala FOSS y se realizó una videofluoroscopia con trago de bario como método diagnóstico confirmatorio tomando los estadios II, III y IV (Tabla 1) de la clasificación de Brombart (21). Los pacientes fueron aleatorizados por bloques, también llama- 
dos bloques permutados, con el fin de asegurar un balance periódico en el número de sujetos asignados a cada a uno de los dos métodos endoscópicos de diverticulotomía (con o sin diverticulotomo); el tiempo del procedimiento se contaba después de la colocación de la sonda en el método estándar o después de colocar el diverticulotomo en posición en el método que usó este accesorio (método con diverticulotomo), ya que es el momento exacto en el cual se inicia el corte del tabique, y se contabilizaba el tiempo (registro en video) cuando este corte terminaba. A continuación se describe detalladamente cómo se realizaba el procedimiento en cada grupo.

\section{Método estándar}

El procedimiento se realiza bajo anestesia general con el paciente en decúbito lateral izquierdo. Un elemento clave es la exposición endoscópica adecuada del tabique que separa el divertículo de la luz esofágica. Esto se logra generalmente mediante la inserción de una sonda nasogástrica (SNG), que se deja en su lugar durante el procedimiento. La SNG protege la pared esofágica contralateral de la lesión térmica durante la sección del tabique. Al endoscopio se le coloca un cap corto y el tabique del divertículo se corta con un papilotomo de punta, al finalizar el corte se coloca un hemoclip en el vértice del corte para prevenir la perforación del mediastino.

\section{Método con diverticulotomo}

Se utiliza un diverticulotomo plástico (Figura 2) diseñado por el Dr. Deviere, el cual tiene dos aletas: una larga que se deja en el esófago y otra corta que se deja en el divertículo y deja expuesto el tabique, el cual se corta con el papilotomo de aguja; no son necesarios ni la SNG ni el cap, y se coloca también el clip al final del procedimiento (Figura 3). El diverticulotomo también puede ser fabricado en una unidad de endoscopia con un sobretubo de colon o de enteroscopia (Figura 4), lo importante a tener en cuenta es que la aleta que se deja en el esófago (larga) mide $3 \mathrm{~cm}$ y la que se deja en el divertículo mide $2 \mathrm{~cm}$.

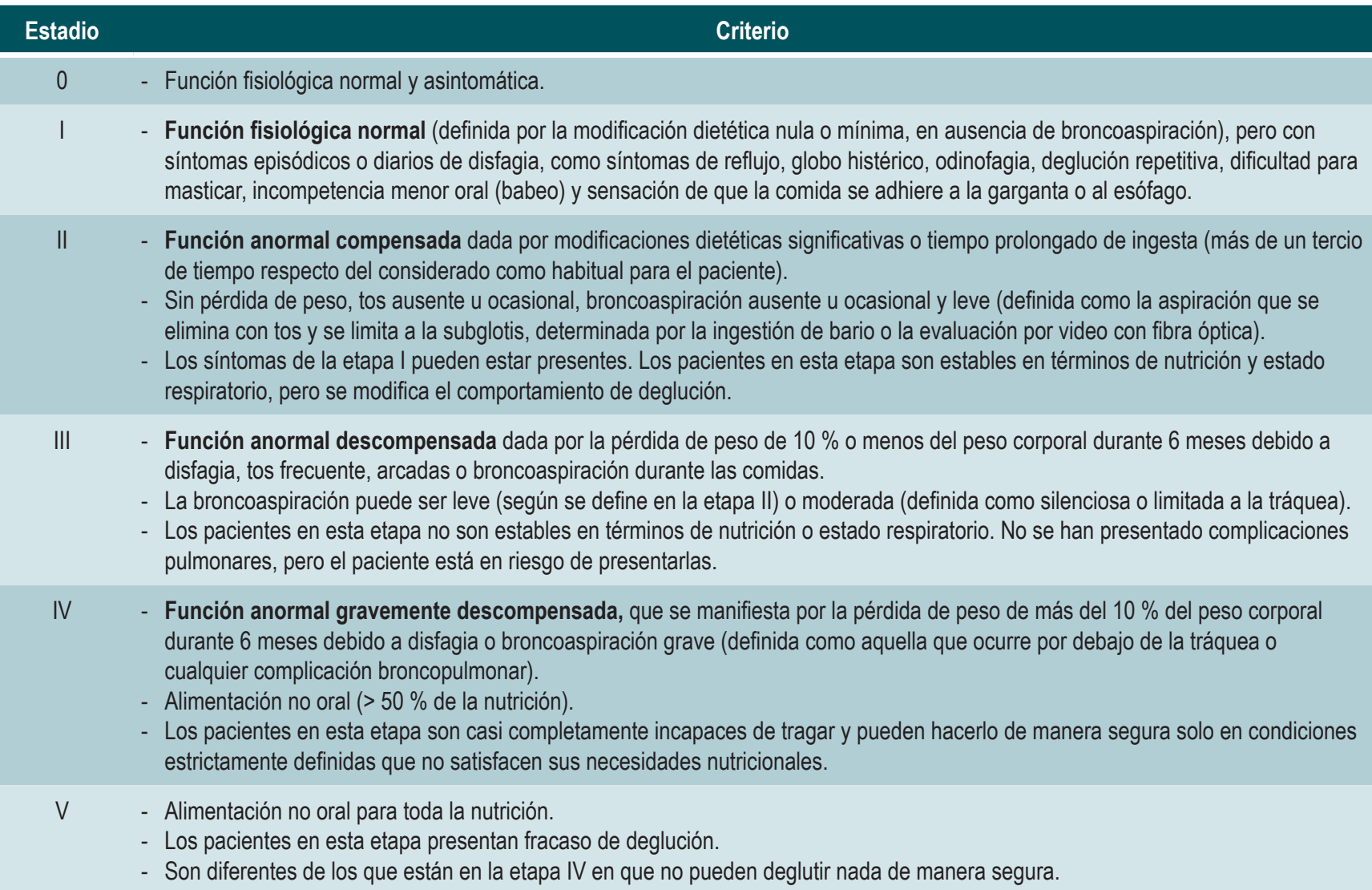

Tomado de: Salassa J. Dig Dis. 1999;17(4):230-4. 


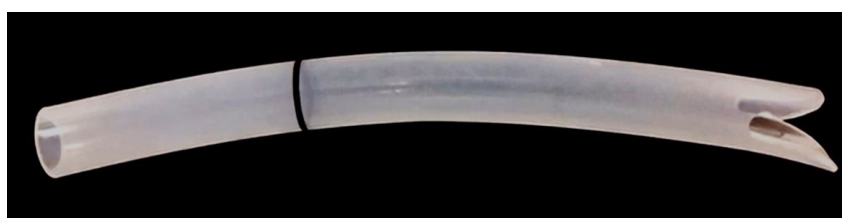

Figura 2. Diverticulotomo diseñado por el Dr. Deviere.

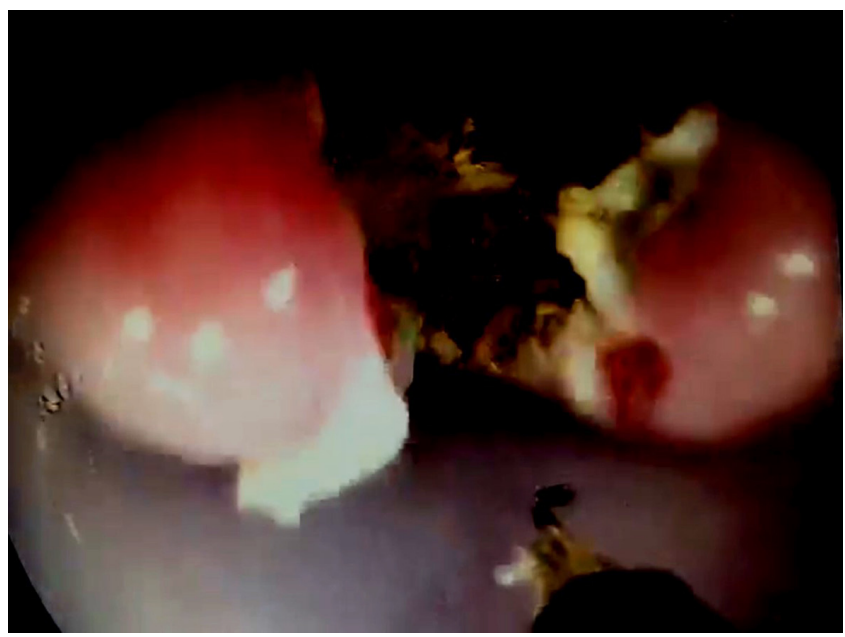

Figura 3. Divertículo de Zenker con el diverticulotomo separando claramente el tabique, el cual se ha cortado con el bisturí de punta.

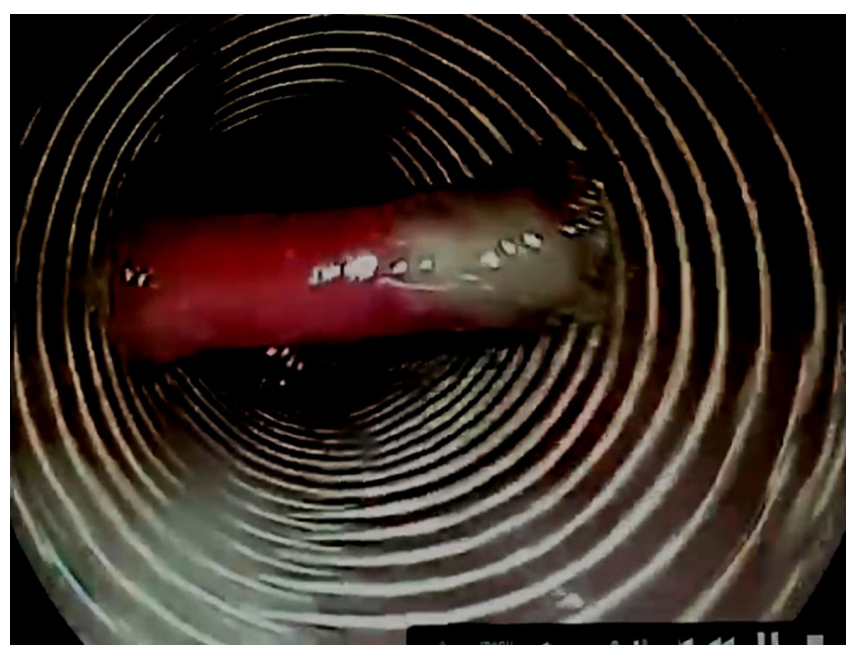

Figura 4. Diverticulotomo diseñado en nuestra unidad.

\section{RESULTADOS}

De enero de 2015 a diciembre de 2018 en total se incluyeron 20 pacientes. Todos tenían como síntoma principal disfagia para sólidos y líquidos. El diagnóstico de Zenker se realizó con una endoscopia inicial y se les realizó un esofagograma para clasificar el tamaño del mismo. El promedio de edad de los pacientes era de 61 años (46-85 años), solo había una mujer (curiosamente la más joven, de 46 años). En cada uno de los grupos se asignaron 10 pacien- tes. El tamaño del divertículo fue en promedio de $3,5 \mathrm{~cm}$ (2,5-7,0; nivel III de la clasificación de Brombart), similar para los dos grupos, se realizó una evaluación con la escala de deglución de resultados funcionales localizándolos en los estadios II-III. En 7 pacientes del grupo del método estándar se resolvieron los síntomas en comparación con 9 del grupo método con diverticulotomo (estadio 0 de la escala de deglución de resultados funcionales, seguimiento mínimo 18 meses). Los tres pacientes que no respondieron presentaron en el seguimiento a 18 meses un estadio II de la escala de deglución de resultados funcionales. Un paciente del grupo del método estándar presentó enfisema como complicación, pero no se evidenció perforación y se manejó de forma conservadora; dos presentaron sangrado que requirió la utilización de la pinza de coagulación con adecuado control. En el método con diverticulotomo no se presentó ninguna complicación, solo un paciente refirió odinofagia a los 15 días y al realizar la endoscopia se encontró que el hemoclip permanecía en su posición y se impactaba en la pared contralateral, por lo cual se retiró y hubo mejoría inmediata. El tiempo promedio del procedimiento fue de 32 (25-45) minutos en el grupo del método estándar y 12 (7-15) minutos en el grupo del método con diverticulotomo $(\mathrm{p}<0,001)$.

\section{DISCUSIÓN}

El divertículo de Zenker ocurre generalmente entre la séptima y octava décadas de la vida y rara vez antes de los 40 años de edad $(6,22)$. La incidencia anual estimada es de 2 por cada 100000 habitantes, su aparición está relacionada con el envejecimiento, ocurre predominantemente en los hombres y se cree que la prevalencia en la población general es del 0,01\% al 0,11\%, que varía según la región: es más común en el norte que en el sur de Europa y tiene mayor frecuencia en los Estados Unidos, Canadá y Australia; y es menos común en Japón e Indonesia. Estos datos solo reflejan a los pacientes sintomáticos, por lo que se desconoce hasta el momento el número de pacientes con divertículo de Zenker asintomáticos $(6,23)$. Los síntomas pueden estar presentes durante semanas o años antes de que se realice el diagnóstico; aunque se ha atribuido una gran cantidad de síntomas, el $80 \%$ a $90 \%$ de los pacientes se quejan de disfagia, además de que los pacientes pueden presentar regurgitación, halitosis, sensación de masa esofágica, tos persistente, odinofagia, carraspeo, y neumonía aspirativa (22). En muchos casos también puede ser un hallazgo incidental, como sucedió en el $17 \%$ de nuestros pacientes, o pueden asistir a urgencias con un cuerpo impactado, como se presentó en el $5 \%$ de nuestros casos (22).

El método diagnóstico más apropiado, dada su amplia disponibilidad, es el esofagograma con trago de bario con 
proyección lateral y visión de área hipofaríngea, el cual permite confirmar el diagnóstico de divertículo de Zenker; sin embargo (en donde esté disponible), se prefiere la fluoroscopia continua dinámica debido a que las imágenes estáticas pueden ser insuficientes en pacientes con divertículos pequeños; además, permite evaluar la presencia de regurgitación y aspiración, y la diferenciación del divertículo de KillianJamieson (ya que aún no está claro si la eficacia y la seguridad del tratamiento endoscópico en estos pacientes son iguales a las de la divertículo de Zenker) (23-25). La clasificación de Brombart permite categorizar el compromiso del divertículo de acuerdo con los hallazgos en este tipo de estudios radiológicos (Tabla 1). Sin embargo, el escenario usual es aquel paciente que refiere disfagia y a quien se le solicita endoscopia de las vías digestivas altas. Es de gran importancia para quienes realizan estos procedimientos tener un alto grado de sospecha de divertículo de Zenker en pacientes adultos mayores que consultan por disfagia, debido a un riesgo elevado de perforación durante el examen (26).

Las medidas terapéuticas para el divertículo de Zenker se describieron desde el siglo XIX. En principio son similares al tratamiento de las hernias, incluidas la movilización y la extirpación del saco diverticular, con o sin cierre del defecto, o inversión endoluminal; más tarde se introdujo la miotomía cricofaríngea (técnica actual). Su abordaje puede ser transoral con autosuturas o más invasivo, mediante cervicotomía lateral con o sin miotomía del cricofaríngeo; sin embargo, este último método presenta complicaciones como mediastinitis, infección del sitio operatorio superficial, parálisis del nervio laríngeo recurrente y fístulas, por lo cual su uso se encuentra en abandono $(27,28)$. La resección quirúrgica tiene como principales desventajas una mayor tasa de complicaciones, como la parálisis del nervio laríngeo recurrente o el hematoma; además, conlleva una mayor duración de la estancia hospitalaria, pero se ha documentado que hasta el $90 \%$ de los pacientes no presenta síntomas después del procedimiento quirúrgico (29).

La evolución del manejo endoscópico del divertículo de Zenker ha sido constante. El pionero fue el Dr. Mosher, en 1917, con el uso del endoscopio rígido y separación del saco de la pared esofágica (presentó complicación por mediastinitis); posteriormente, en 1932 la técnica fue modificada por Seiffert, aunque son Dohlman y Matson quienes llevaron a cabo de forma exitosa diverticulotomías a través de visión endoscópica; en 1982 Van Overbeek introdujo el uso del láser de dióxido de carbono $\left(\mathrm{CO}_{2}\right)$ a la técnica y en 1993, de forma independiente, Collard y colaboradores, en Bélgica, y Martin-Hirsch y Newbegin, en el Reino Unido, describieron la diverticulotomía mediante el uso de suturas mecánicas $(28,30,31)$. Las principales ventajas de la técnica endoscópica flexible, en comparación con las opciones de tratamiento quirúrgico, es que es más sencilla, tiene una menor estancia hospitalaria (llegando alguna incluso a ser ambulatoria), la tasa general de éxito es hasta del $90 \%$ y la recurrencia de síntomas aparece solo en el 10,5\% de los pacientes (32). Las complicaciones generales y la tasa de mortalidad por diverticulotomía de Zenker endoscópica flexible es de $15 \%$ y $0 \%$, respectivamente, y las complicaciones más frecuentes son el enfisema cervical $(5,7 \%$, no necesita manejo si no hay extravasación de medio), la perforación $(4,0 \%$, usualmente se resuelve con la colocación de clips) y el sangrado (3,1\%, usualmente se controla en el mismo procedimiento) (33-36).

Mas recientemente, se ha descrito el uso de miotomía endoscópica peroral (POEM), en el manejo del divertículo de Zenker (Z-POEM), con una tasa de éxito clínico del $92 \%$ y una tasa de perforación del 5,5\% (37). Esta técnica ha ganado popularidad en los últimos años y la ventaja potencial sobre la septotomía endoscópica estándar es la división completa del tabique, lo que disminuye el riesgo de recurrencia de los síntomas. Sin embargo, se debe tener en cuenta que en la recurrencia posterior al Z-POEM (teóricamente menor), la estrategia de manejo aún no está clara, especialmente debido a la probabilidad de fibrosis submucosa posterior. Estos aspectos se deben tener en cuenta cuando se seleccionan los pacientes; sin embargo, esta técnica tiene un papel protagónico y crucial en la actualidad y en el futuro cercano $(37,38)$.

En la Tabla 4 se muestran las series endoscópicas publicadas hasta ahora en comparación con la nuestra. Cabe anotar que nuestra serie es pequeña, pero significativa para nuestro medio. A pesar de esto, el éxito del procedimiento y la baja tasa de complicaciones coinciden con lo reportado en la actualidad. Se debe mencionar que, aunque muchos autores realizan este procedimiento endoscópico bajo sedación, consideramos que siempre debería realizarse bajo anestesia general ya que es mejor tener al paciente totalmente inmóvil y existe el riesgo potencial de sangrado, por lo que se recomienda proteger la vía aérea debido al riesgo de brocoaspiración, el cual se puede minimizar con el uso en la fase inicial de corriente de coagulación en la unidad electroquirúrgica. Otra medida útil es el uso de un bisturí endoscópico (gancho de disección, triángulo, punta de cerámica, entre otros) más que el papilotomo de precorte porque este es muy delgado y con frecuencia se adhiere el tejido y obliga a su retiro y reinserción frecuente para la realización de limpieza, un truco adicional es la utilización del papilotomo de punta de cerámica en la fase inicial para apoyar en el tabique y prevenir la perforación de la pared del esófago.

Como muestra, nuestro estudio sobre la experiencia con la diverticulotomía endoscópica con diverticulotomo ha sido altamente satisfactoria en términos de menor duración 
Tabla 4. Experiencia con diverticulotomía endoscópica. Reporte de grupos de trabajo

\begin{tabular}{|c|c|c|c|c|c|c|c|c|c|}
\hline \multirow[t]{2}{*}{ Primer autor } & \multirow[t]{2}{*}{ Año } & \multirow[t]{2}{*}{$\mathbf{n}$} & \multirow{2}{*}{$\begin{array}{l}\text { Dieta POP, } \\
\text { hora }\end{array}$} & \multicolumn{2}{|c|}{ Complicaciones } & \multicolumn{2}{|c|}{ Recurrencia } & \multirow{2}{*}{$\begin{array}{c}\text { Estancia hospitalaria, } \\
\text { horas }\end{array}$} & \multirow[t]{2}{*}{ Seguimiento } \\
\hline & & & & Número & $\%$ & $\mathbf{N}$ & $\%$ & & \\
\hline Ishioka (41) & 1995 & 42 & NA & $2 / 42$ & 5 & $3 / 42$ & 7,1 & NA & 38 meses \\
\hline Hashiba (42) & 1999 & 47 & 24 & $7 / 47$ & 14 & $2 / 47$ & 4 & Ambulatorio & 1 día a 1 año \\
\hline Sakai (43) & 2001 & 10 & $48 \mathrm{~h}$ & 0 & NA & 0 & NA & NA & 2 a 12 meses \\
\hline de la Morena (44) & 2005 & 3 & $48 \mathrm{~h}$ & 0 & NA & 0 & NA & 48 & 15 meses \\
\hline Vogelsang (45) & 2006 & 31 & NA & $7 / 31$ & 23 & $10 / 31$ & 32 & NA & 26 meses \\
\hline Costamagna (46) & 2007 & 39 & NA & $9 / 39$ & 23 & $9 / 39$ & 23 & $72 \mathrm{~h}$ & 36 meses \\
\hline Al Kadi (3) & 2010 & 18 & $24 \mathrm{~h}$ & $1 / 18$ & 6 & $2 / 18$ & 11 & $24-48 h$ & 27,5 meses \\
\hline Gómez (4) & 2010 & 9 & $12 \mathrm{~h}$ & $1 / 9$ & 11 & $1 / 9$ & 11 & $24 \mathrm{~h}$ & 25 meses \\
\hline Gómez & 2018 & 20 & $12 \mathrm{~h}$ & $2: 20$ & 10 & 2 & 10 & $24 \mathrm{~h}$ & 18 meses \\
\hline
\end{tabular}

del procedimiento, lo que en el contexto de este grupo de pacientes, que en general se consideran de alto riesgo quirúrgico (por la edad, comorbilidades, estado nutricional y una mayor fragilidad), hace una diferencia significativa en la evolución posoperatoria del paciente, sin afectar los resultados en términos de reaparición de síntomas (39, $40)$. El tiempo promedio del procedimiento fue de 32 (2545) minutos en el grupo del método estándar y de solo 12 (7-15) minutos en el grupo del método con diverticulotomo $(p<0,001)$, sin diferencias significativas en complicaciones o recurrencia, y comparable a las series publicadas en la literatura. Sin embargo, su seguridad comprobada debe evaluarse prospectivamente en estudios posteriores con una mayor cantidad de procedimientos.

\section{CONCLUSIÓN}

La miotomía del divertículo de Zenker con el uso de endoscopia flexible utilizando el diverticulotomo tiene beneficios potenciales en términos de tiempos operatorios y estancias posoperatorias más cortas y un inicio más temprano de la dieta. Desde el punto de vista técnico, permite una mejor exposición del tabique, lo cual facilita su corte, aunque se necesitan estudios más grandes para confirmar estos resultados.

\section{REFERENCIAS}

1. Tabola R, Lewandowski A, Cirocchi R, Augoff K, Kozminska U, Grabowski K. Zenker diverticulum: Experience in surgical treatment of large diverticula. Medicine (Baltimore). 2018;97(19):e0557. https://doi.org/10.1097/MD.0000000000010557

2. Visser LJ, Hardillo JA, Monserez DA, Wieringa MH, Baatenburg de Jong RJ. Zenker's diverticulum: Rotterdam experience. Eur Arch Otorhinolaryngol. 2016;273(9):2755-63. https://doi.org/10.1007/s00405-015-3825-0

3. Al-Kadi A, Maghrabi A, Thomson D, MGillman L, Dhalla S. Endoscopic Treatment of Zenker. Diverticulum: Results of a 7-Year Experience. J Am Coll Surg. 2010;211(2):239-243. https://doi.org/10.1016/j.jamcollsurg.2010.04.011
4. Gómez Z MA, Ardila SF, Arbeláez V. Experiencia en el manejo del divertículo de Zenker: Una serie de 18 casos. Rev Col Gastroenterol. 2011;26(2):100-105.

5. Ishaq S, Sultan H, Siau K, Kuwai T, Mulder CJ, Neumann $H$. New and emerging techniques for endoscopic treatment of Zenker's diverticulum: State-of-the-art review. Dig Endosc. 2018;30(4):449-460. https://doi.org/10.1111/den.13035

6. Law R, Katzka DA, Baron TH. Zenker's Diverticulum. Clinical Gastroenterology and Hepatology. 2014;12(11):1773-1782. https://doi.org/10.1016/j.cgh.2013.09.016

7. Ciuc D, Birlă R, Panaitescu E, Tanțău M, Constantinoiu S. Zenker Diverticulum Treatment: Endoscopic or Surgical? 
Chirurgia (Bucur). 2018;113(2):234-243.

https://doi.org/10.21614/chirurgia.113.2.234

8. Jones D, Aloraini A, Gowing S, Cools-Lartigue J, Leimanis M, Tabah R, Ferri L. Evolving Management of Zenker's Diverticulum in the Endoscopic Era: A North American Experience. World J Surg. 2016;40(6):1390-6. https://doi.org/10.1007/s00268-016-3442-0

9. Prisman E, Genden EM. Zenker diverticulum. Otolaryngol Clin North Am. 2013;46(6):1101-11. https://doi.org/10.1016/j.otc.2013.08.011

10. Henry MA, Lerco MM, Tagliarini JV, Castilho EC, Novaes FT, Lamonica VC. Pharyngoesophageal diverticulum: evaluation of treatment results. Rev Col Bras Cir. 2013;40(2):104-9. https://doi.org/10.1590/S0100-69912013000200004

11. Aghajanzadeh M, Yousefi-Mashhoor M, Delshad MAE, Massahnia S. Zenker's Diverticulum: Report Rare Presentation and Management of Six Cases. Gastroenterol Hepatol Open Access 2016, 4(6): 00124. https://doi.org/10.15406/ghoa.2016.04.00124

12. Stewart, KE, Smith DRK, Woolley SL. Simultaneously occurring Zenker's diverticulum and Killian-Jamieson diverticulum: case report and literature review. Gastroenterol Hepatol Open Access. 2017;131(8),661-666. https://doi.org/10.1017/S0022215117001268

13. Dina I, Ginghina $O$, Toderescu CD, Bălălău C, Galateanu B, Negrei C, Iacobescu C. Zenker's diverticulum and squamous esophageal cancer: a case report. J Mind Med Sci. 2017;4(2):193-197. https://doi.org/10.22543/7674.42.P193197

14. Mantsopoulos K, Psychogios G, Karatzanis A, Künzel J, Lell M, Zenk J, Koch M. Clinical relevance and prognostic value of radiographic findings in Zenker's diverticulum. Eur Arch Otorhinolaryngol. 2014;271(3):583-8. https://doi.org/10.1007/s00405-013-2562-5

15. Aiolfi A, Scolari F, Saino G, Bonavina L. Current status of minimally invasive endoscopic management for Zenker diverticulum. World J Gastrointest Endosc 2015;7(2):87-93. https://doi.org/10.4253/wjge.v7.i2.87

16. Albers DV, Kondo A, Bernardo WM, Sakai P, Moura RN, Silva GL, Ide E, Tomishige T, de Moura EG. Endoscopic versus surgical approach in the treatment of Zenker's diverticulum: systematic review and meta-analysis. Endosc Int Open. 2016;4(6):E678-E686. https://doi.org/10.1055/s-0042-106203

17. Bloom JD, Bleier BS, Mirza N, Chalian AA, Thaler ER. Factors predicting endoscopic exposure of Zenker's diverticulum. Ann Otol Rhinol Laryngol. 2010;119(11):736-41. https://doi.org/10.1177/000348941011901116

18. Saetti R, Silvestrini M, Peracchia A, Narne S. Endoscopic stapler-assisted Zenker's diverticulotomy: which is the best operative facility? Head Neck. 2006;28(12):1084-9. https://doi.org/10.1002/hed.20431

19. Salassa J. A Functional Outcome Swallowing Scale for Staging Oropharyngeal Dysphagia. Dig Dis.
1999; 17(4):230-4. https://doi.org/10.1159/000016941

20. Bergeron JL, Long JL, Chhetri DK. Dysphagia Characteristics in Zenker's Diverticulum. Otolaryngol Head Neck Surg. 2013;148(2): 223-228. https://doi.org/10.1177/0194599812465726

21. Miller FR, Taylor C. Zenker's Diverticulum: Endoscopic Surgical Management Options. Clin Surg. 2017;2:1422.

22. Ferreira LE, Simmons DT, Baron TH. Zenker's diverticula: pathophysiology, clinical presentation, and flexible endoscopic management. Dis Esophagus. 2008;21(1):1-8. https://doi.org/10.1111/j.1442-2050.2007.00795.x

23. Bizzotto A, Iacopini F, Landi R, Costamagna G. Zenker's diverticulum: exploring treatment options. Acta Otorhinolaryngol Ital. 2013;33(4):219-29.

24. Naprawa G, Białkowska J. Foreign body in a Zenker's diverticulum (looking for the lost dentures). Prz Gastroenterol. 2014;9(4):254-8. https://doi.org/10.5114/pg.2014.45109

25. Wong HJ, Ujiki MB. Per Oral Zenker Diverticulotomy. Surg Clin North Am. 2020;100(6):1215-1226. https://doi.org/10.1016/j.suc.2020.08.005

26. Babür, T. Iatrogenic Zenker's diverticulum perforation: A conservatively treated case. Ulus Cerrahi Derg. 2013;30(4):234-6. https://doi.org/10.5152/UCD.2013.24

27. Rizzetto C, Zaninotto G, Costantini M, Bottin R, Finotti E, Zanatta L, Guirroli E, Ceolin M, Nicoletti L, Ruol A, Ancona E. Zenker's diverticula: feasibility of a tailored approach based on diverticulum size. Journal of Gastrointestinal Surgery, 2008;12(12), 2057-2065. https://doi.org/10.1007/s11605-008-0684-7

28. Sakai P. Endoscopic myotomy of Zenker's diverticulum: lessons from 3 decades of experience. Gastrointestinal endoscopy. 2016;83(4):774-5. https://doi.org/10.1016/j.gie.2015.10.031

29. Verdonck J, Morton RP. Systematic review on treatment of Zenker's diverticulum. Eur Arch Otorhinolaryngol. 2015;272(11):3095-107. https://doi.org/10.1007/s00405-014-3267-0

30. Battaglia G, Antonello A, Realdon S, Cesarotto M, Zanatta L, Ishaq S. Flexible endoscopic treatment for Zenker's diverticulum with the SB Knife. Preliminary results from a single-center experience. Dig Endosc. 2015;27(7):728-33. https://doi.org/10.1111/den.12490

31. Nielsen HU, Trolle W, Rubek N, Homøe P. New technique using LigaSure for endoscopic mucomyotomy of Zenker's diverticulum: diverticulotomy made easier. Laryngoscope. 2014;124(9):2039-42. https://doi.org/10.1002/lary.24558

32. Ishaq S, Hassan C, Antonello A, Tanner K, Bellisario C, Battaglia G, Anderloni A, Correale L, Sharma P, Baron $\mathrm{TH}$, Repici A. Flexible endoscopic treatment for Zenker's diverticulum: a systematic review and meta-analysis. Gastrointest Endosc. 2016;83(6):1076-1089.e5. https://doi.org/10.1016/j.gie.2016.01.039 
33. Wilmsen J, Baumbach R, Stüker D, Weingart V, Neser F, Gölder SK, Pfundstein C, Nötzel EC, Rösch T, Faiss S. New flexible endoscopic controlled stapler technique for the treatment of Zenker's diverticulum: A case series. World J Gastroenterol. 2017;23(17):3084-3091. https://doi.org/10.3748/wjg.v23.i17.3084

34. Yuan Y, Zhao YF, Hu Y, Chen LQ. Surgical treatment of Zenker's diverticulum. Dig Surg. 2013;30(3):207-18. https://doi.org/10.1159/000351433

35. Fan HS, Stavert B, Chan DL, Talbot ML. Management of Zenker's diverticulum using flexible endoscopy. VideoGIE. 2019;4(2):87-90. https://doi.org/10.1016/j.vgie.2018.12.007

36. Pescarus R, Shlomovitz E, Sharata AM, Cassera MA, Reavis KM, Dunst CM, Swanström LL. Trans-oral cricomyotomy using a flexible endoscope: technique and clinical outcomes. Surg Endosc. 2016;30(5):1784-9. https://doi.org/10.1007/s00464-015-4445-x

37. Yang J, Novak S, Ujiki M, Hernández Ó, Desai P, Benias P, Lee D, Chang K, Brieau B, Barret M, Kumta N, Zeng X, Hu B, Delis K, Khashab MA. An international study on the use of peroral endoscopic myotomy in the management of Zenker $>$ s diverticulum. Gastrointest Endosc. 2020;91(1):163-168. https://doi.org/10.1016/j.gie.2019.04.249

38. Ishaq S, Kuwai T, Siau K, Mulder CJ, Neumann H. Is Z-POEM for Zenker $>$ s the same as POEM for achalasia? Or we are barking up the wrong tree? Gastrointest Endosc. 2020;91(1):204-205. https://doi.org/10.1016/j.gie.2019.07.028

39. Ko FC. Preoperative Frailty Evaluation: A Promising Riskstratification Tool in Older Adults Undergoing General Surgery. Clin Ther. 2019;41(3):387-399. https://doi.org/10.1016/j.clinthera.2019.01.014

40. Shinall MC Jr, Arya S, Youk A, Varley P, Shah R, Massarweh NN, Shireman PK, Johanning JM, Brown AJ, Christie NA, Crist L, Curtin CM, Drolet BC, Dhupar R,
Griffin J, Ibinson JW, Johnson JT, Kinney S, LaGrange

C, Langerman A, Loyd GE, Mady LJ, Mott MP, Patri

M, Siebler JC, Stimson CJ, Thorell WE, Vincent SA, Hall DE. Association of Preoperative Patient Frailty and Operative Stress With Postoperative Mortality. JAMA Surg. 2019; 155(1):e194620. https://doi.org/10.1001/jamasurg.2019.4620

41. Ishioka S, Sakai P, Maluf Filho F, Melo JM. Endoscopic incision of Zenker's diverticula. Endoscopy. 1995;27(6):433-7. https://doi.org/10.1055/s-2007-1005736

42. Hashiba K, de Paula AL, da Silva JG, Cappellanes CA, Moribe D, Castillo CF, Brasil HA. Endoscopic treatment of Zenker's diverticulum. Gastrointest Endosc. 1999;49(1):93-7. https://doi.org/10.1016/s0016-5107(99)70452-9

43. Sakai P, Ishioka S, Maluf-Filho F, Chaves D, Moura EG. Endoscopic treatment of Zenker's diverticulum with an oblique-end hood attached to the endoscope. Gastrointest Endosc. 2001;54(6):760-3. https://doi.org/10.1067/mge.2001.119606

44. de la Morena E, Pérez-Arellano E, Carreño R, Tomás E, González-Lama Y. Tratamiento endoscópico del divertículo de Zenker [Endoscopic treatment of Zenker's diverticulum]. Cir Esp. 2005;78(4):256-9.

45. Vogelsang A, Preiss C, Neuhaus H, Schumacher B. Endotherapy of Zenker's diverticulum using the needleknife technique: long-term follow-up. Endoscopy. 2007;39(2):131-6. https://doi.org/10.1055/s-2006-944657

46. Costamagna G, Iacopini F, Tringali A, Marchese M, Spada C, Familiari P, Mutignani M, Bella A. Flexible endoscopic Zenker's diverticulotomy: cap-assisted technique vs. diverticuloscope-assisted technique. Endoscopy. 2007;39(2):146-52. https://doi.org/10.1055/s-2007-966140 